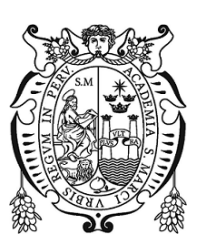

Revista de Investigación de Física 24(1), (Ene-Jun 2021)

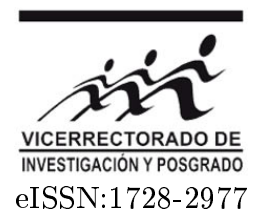

\title{
Análisis de la percepción de los estudiantes ante la enseñanza virtual de las Ciencias Físicas en la Universidad Nacional Mayor de San Marcos
}

\author{
Miguel Castillo ${ }^{*}$, Marco Merma ${ }^{1}$, Juan Mendoza ${ }^{1}$, José Janampa $^{2}$, Glissett Mendoza ${ }^{3}$ y Nadia Rea ${ }^{4}$ \\ ${ }^{1}$ Universidad Nacional Mayor de San Marcos, Facultad de Ciencias Físicas, Lima, Perú \\ ${ }^{2}$ Universidad Nacional Mayor de San Marcos, Facultad de Medicina Humana, Lima, Perú \\ ${ }^{3}$ Universidad Nacional de Barranca, Dpto. Académico de Ciencias Básicas y Afines, Barranca, Perú \\ ${ }^{4}$ Universidad Nacional José Faustino Sánchez Carrión, Huacho, Perú
}

Recibido 05 febrero 2021 - Aceptado 30 abril 2021

\begin{abstract}
Resumen
El presente trabajo, nos ha permitido procesar y analizar los datos obtenidos acerca de la percepción de los estudiantes ante la enseñanza virtual de las ciencias físicas en el sistema académico de formación universitaria en esta etapa de pandemia, producto de la Covid-19; el sistema de enseñanza en virtud de la emergencia sanitaria se cambió en forma brusca de la modalidad de educación presencial clásica a una nueva modalidad de educación virtual. Para el presente trabajo fueron encuestados alrededor de 100 estudiantes de las facultades de ciencias básicas como Ciencias Físicas y Ciencias Biológicas, además de la Escuela Profesional de Educación y la Escuela Profesional de Ingeniería Eléctrica de la Universidad Nacional Mayor de San Marcos, esta encuesta fue realizada al concluir el semestre 2020-I. Se aplicó un formulario de encuesta con 16 preguntas para ser respondidas por los estudiantes, dichas preguntas eran con respecto a:

1.- Las capacitaciones, equipos técnicos e internet.

2.- Los docentes y su metodología aplicada.

3.- Los recursos didácticos.

De los resultados obtenidos sobre: capacitaciones, equipos técnicos e internet un gran porcentaje (59.6\%) de los estudiantes encuestados manifestaron que no necesitan de las capacitaciones porque conocen el tema de aulas virtuales y sus herramientas digitales, la mayoría de los estudiantes encuestados $(67 \%)$ manifiestan tener sus propios equipos electrónicos computacionales para desarrollar sus clases virtuales. Sobre los docentes y su metodología, los alumnos manifiestaron que fue buena (31.9\%); frente al sistema de evaluación han señalado que fue buena (54.3\%). Respecto a los docentes y su metodología aplicada, los estudiantes manifestaron su satisfacción (37.2\%), además calificaron al docente de regular $(30.9 \%)$ durante esta etapa de las clases virtuales. Con respecto a los recursos didácticos, los estudiantes mayoritariamente manifestaron en un $75.5 \%$ que han utilizado recursos didácticos, un grupo representativo de estudiantes (43.6\%) manifestaron que los profesores dominan el uso de los recursos y materiales digitales para las clases virtuales, asi como un grupo mayoritario de estudiantes $(51.1 \%)$ manifestaron que los recursos y materiales empleados por los profesores han servido para la enseñanza-aprendizaje de las clases virtuales.
\end{abstract}

Palabras clave: Covid-19, educación virtual, recursos didácticos.

Analysis of students' perception of virtual teaching of Physical Sciences at the Universidad Nacional Mayor de San Marcos

*mcastilloc@unmsm.edu.pe 


\begin{abstract}
This work, has allowed us to process and analyze the data obtained about the perception of students in the face of the virtual teaching of physical sciences in the academic system of university training at this stage of pandemic, product of the Covid-19; the system of education under the health emergency was abruptly changed from the modality of classical face-to-face education to a new mode of virtual education. For this work, around 100 students were surveyed basic science faculties such as Physical Sciences and Biological Sciences, as well as the Professional School of Education and the Professional School of Education and the Professional School of Electrical Engineering of the National University of San Marcos, this survey was conducted at the end of the 2020-I semester.

A survey form was applied with 16 questions to be answered by students, those questions were regarding:

1.- Training, technical equipment and the internet.

2.- Teachers and their methodology applied.

3.- Teaching resources.

Of the results obtained on: trainings, technical teams and the internet a large percentage $(59.6 \%)$ of the students surveyed stated that they do not need the trainings because they know the topic of virtual classrooms and their digital tools, most students surveyed (67\%) they manifest themselves to have their own computer electronic equipment to develop their virtual classes. On teachers and their methodology, students said it was good (31.9\%); in the face of the evaluation system they have pointed out that it was also good $(54.3 \%)$. About the teachers and their applied methodology, the students expressed their satisfaction (37.2\%), they also rated the teacher as regular (30.9\%) during this stage of the virtual classes. About didactic resources, $75.5 \%$ of students stated that they had used didactic resources, a representative group of students (43.6\%) stated that teachers dominate the use of digital resources and materials for virtual classes, as well as A majority group of students (51.1\%) stated that the resources and materials used by the teachers have served for the teaching-learning of virtual classes.
\end{abstract}

Keywords: Covid-19, virtual education, didactic resources.

\section{Introducción}

La crisis sanitaria ocasionada por la Covid-19 en todo el planeta, ha hecho que nuestro país se vea afectado seriamente, desnudando los problemas en los sectores más importantes como son salud, educación y trabajo.

El sector salud ha sido afectado seriamente, sumado a ello las carencias de camas para internamientos, camas para la Unidad de Cuidados Intensivos (UCI), falta de oxígeno medicinal y medicamentos, que permitan enfrentar la pandemia para los más de 32 millones de peruanos.

En el sector de educación, no hemos tenido capacidad tecnológica para enfrentar el cambio de la educación presencial a la educación virtual en todos los niveles de la educación, incluso en la educación superior universitaria. A pesar de que el poder ejecutivo ha destinado presupuestos de emergencias para enfrentar la covid-19, en el sector educación y las universidades públicas, lamentablemente la burocracia del sector público hace lento el destino de este presupuesto para los más necesitados como son los alumnos y profesores de las universidades públicas.

En este contexto la Universidad Nacional Mayor de San Marcos tuvo que iniciar sus actividades académicas (semestre 2020-I) el lunes 08 de junio, y culminar dichas actividades a fines de setiembre del 2020. Se aplicó un formulario encuesta aproximadamente a 100 estudiantes de las facultades como: Ciencia Físicas, Ciencias Biológicas y la Escuela Profesional (EP) de Ing Eléctrica y la EP de Educación. Los resultados han revelado algunos indicadores muy importantes para comprender la real dimensión en la percepción de los estudiantes ante la enseñanza virtual de las Ciencias Físicas, en cómo los ha afectado en la enseñanza, sobre todo en didáctica, metodología, recursos didácticos y tecnológicos.

\section{Contexto externo:}

1. Crisis Sanitaria.- Según datos del Ministerio de Salud (MINSA), antes del inicio de la pandemia, en marzo del 2020, en nuestro país teníamos 276 camas para la Unidad de Cuidados Intensivos (UCI) y menos de 750 camas para hospitalización a nivel nacional para una población de más de 32 millones de peruanos, sumado a ello la carencia de oxígeno medicinal, lo poco que había en los hospitales no abastecían la necesidad de los pacientes afectados por la Covid-19.

2. Crisis Sistema Educativo.- Ante el cambio del sistema de la Educación Presencial a una nueva Educación Virtual o remota, el sistema educativo nacio- 
nal, no estaba preparado para este tipo de educación, ni los estudiantes, ni los profesores. Se necesitaba equipamientos informáticos para todos ellos. El Ministerio de Educación (MINEDU) tuvo que utilizar el canal de televisión y la radio estatal; además otros medios de radio, televisión regional y local para desarrollar la educacion virtual a través del programa "Aprendo en Casa" en la Educación Básica Regular.

Algo similar ocurría a nivel de la Educación Superior, no estábamos preparados con equipamientos informáticos y herramientas digitales, ni los estudiantes ni los profesores universitarios, además los sílabos tenían que ser adaptados a la nueva forma de educación virtual.

3. Crisis Económica.- La crisis sanitaria producida por la Covid-19, ha causado una enorme recesión en los sectores principalmente informales de la economía nacional, muchas pequeñas empresas han cerrado o han dejado de producir, y aún continua la recesión para los sectores más pobres; sin embargo, se han aperturado los grandes negocios, como las grandes cadenas de restaurantes y centros comerciales. Estos negocios se constituyen en altamente peligroso por la proliferación de posibles amenazas de contagios sumado a ello el negocio del transporte interprovincial y el transporte aéreo. El gobierno ha implementado bonos como medida de apoyo a los más necesitados, sin embargo por los principales medios de comunicación del país, hemos visto denuncias que dichas ayudas nunca llegaron a la población mas necesitada.

La gran lección de esta pandemia, es que los sectores más pobres y vulnerados son los más golpeados, mientras que los más favorecidos han sido los grupos de poder económico del país.

A estos grupos de poder, les interesa más sus negocios y producción que la vida y la salud de la población, se conoce que el sector de los empresarios han presionado al poder ejecutivo para la apertura de sus negocios en nuestro país, que en parte lo han logrado con la reactivación económica de la fase 3.

\section{Contexto interno:}

1. En equipamiento: La Universidad Nacional Mayor de San Marcos, reinició sus actividades académicas la primera semana de junio del 2020, muy a pesar de no haberse implementado ningún tipo de ayuda a los docentes, como por ejemplo: para los gastos de fluido eléctrico, de internet y de equipos computacionales; todos estos han sido asumidos por los propios profesores universitarios, en medio de las crisis sanitarias de la pandemia. A finales de ciclo, la Universidad ha brindado de equipo un modem de ayuda para el uso de los docentes, porque el ejecutivo había destinado presupuesto econónomico adicional para enfrentar la educación virtual en medio de la crisis sanitaria generada por la Covid19.

2. En Salud de los Docentes: Los profesores sanmarquinos, se han visto afectados seriamente, si bien es cierto que el docente universitario dicta un promedio de 12 horas semanales, en esta pandemia muchos permanecen sentados varias horas para realizar actividades como: preparación de clases, clases virtuales, elaboración de materiales, revisión de prácticas y hasta reuniones virtuales, como consecuencia generan problemas en la salud de los profesores.

Los problemas que han surgido producto del uso excesivo de la computadora y los celulares son: de la visión, de la columna vertebral y de la circulación sanguínea producto de la mala posición y de estar sentado durante horas, ademas el sedentarismo por la falta de movimiento y actividades de los docentes universitarios; el problema del estrés es un daño adicional en la salud mental de los profesores universitarios.

3. Sistema de Enseñanza y Evaluación: El sistema de enseñanza ha cambiado drásticamente de una forma presencial a una forma remota y virtual, algo que nunca había sucedido con nuestro sistema educativo nacional, en ninguno de sus niveles, por lo que nos cogió desprevenidos. En esa situación la universidad medianamente desarrolló una serie de capacitaciones para el uso de herramientas digitales, para enseñar a los docentes a enfrentar la situación de la nueva educación, pero que se veía seriamente afectado con las carencias de equipos, de herramientas que acompañan las clases, como: softwares y simuladores, recursos de apoyo para las clases. Al terminar el ciclo 2020-I, hemos logrado atender las necesidades de los alumnos, dentro del contexto de la virtualidad, con ciertas dificultades en la adecuación a la nueva educación, carencia de herramientas, softwares, metodologías y didácticas en esta nueva etapa de la era de la educación virtual. Finalmente, el sistema de evaluación aplicado, es el mismo empleado en la modalidad presencial, no existe forma alguna en la educación virtual que garantice el desarrollar de los exámenes. Por lo que se propuso que los pesos de las evaluaciones en esta etapa de la nueva educación virtual cambiara y se diera mayor peso a las actividades procedimentales desarrolladas en el aula en cada clase, es decir dar 
mayor peso a las participaciones progresivas de los alumnos durante el desarrollo las clases virtuales.

4. Situación de los Estudiantes: Muchos estudiantes carecen de recursos y equipos computacionales, y esta pandemia los ha golpeado a nivel económico, en equipamientos y de salud. Según datos del Ministerio de Educación (MINEDU) unos 174.000 estudiantes universitarios que equivale $18.6 \%$ del total de estudiantes universitarios del país han dejado de estudiar por motivos principalmente de la Covid-19, del cual $9.85 \%$ son de las Universidades públicas, mientras que $22.5 \%$ son de las Universidades privadas (fuente MINEDU-Setiembre 2020). El estado para enfrentar y contener las consecuencias y sus efectos de la pandemia Covid-19 en el sistema universitario ha invertido más de 600 millones de soles. Específicamente para ayudar en la conectividad de los estudiantes universitarios, se han invertido 30.8 millones para la compra y distribución de chips para la conectividad a internet y poder llevar sus clases virtuales (fuente MINEDU-Setiembre 2020). Para la Universidad Nacional Mayor de San Marcos fue asignado un presupuesto de $S / 2011.400$ para brindar ayuda de chips para la conectividad a una población de 2345 alumnos beneficiarios, de un total de aproximadamente de 30 mil estudiantes, lo que equivale $7.8 \%$ del total de alumnos (Fuente MINEDU-Setiembre 2020).

\section{Conceptos básicos}

El cambio de la educación presencial a la nueva educación virtual, producto de la pandemia originado por la Covid-19, ha producido cambios y transformaciones inmediatas, generando una profunda preocupación, dificultades entre los docentes de la Universidad Nacional Mayor de San Marcos. Los cambios no planificados desataron una profunda preocupación, ante tal situación la Universidad medianamente capacitó a sus docentes para esta nueva era de la educación virtual, mediante una series de cursos como: Entornos Virtuales de Aprendizaje de Google (EVA-G) y Contenidos Educativos Digitales de Google (CED-G). Pero que cada docente en forma personal debia de explorar y utilizar simuladores, programas o softwares para reforzar sus clases virtuales, en las áreas de ciencias básicas se emplearon programas y simuladores de laboratorios.

Dentro de la enseñanza virtual en esta etapa de pandemia, especialmente para los cursos de ciencias básicas, en este caso específico los cursos de Física, la tarea no fue nada fácil, por la naturaleza de los cursos de la carrera, éstos siempre llevan la parte práctica y sus laboratorios en el modo presencial; para este nuevo trabajo remoto, se tuvieron que adecuar y adaptar con prácticas en lí- nea y simuladores apropiados, tal como se trata a modo de referencia en el párrafo siguiente del artículo de Laboratorios de enseñanza durante una pandemia: "Lecciones de la primavera de 2020 y perspectivas para el futuro, menciona respecto a las Actividades de laboratorio. Se adoptaron una variedad de enfoques al realizar la transición a laboratorios remotos, siendo los más comunes: proporcionar a los estudiantes datos para analizar; realización de actividades de laboratorio mediante simulaciones; hacer que los estudiantes vean videos del instructor o asistente técnico que dirige el laboratorio; y completar experimentos en casa con equipo doméstico o equipo enviado por el instructor. En esta sección, discutimos los 7 tipos principales de actividades utilizadas en orden del más frecuentemente reportado en la encuesta de instructores. Terminamos la sección con una discusión sobre cómo los instructores utilizaron la escritura como un elemento importante de las clases de laboratorio remoto" [Fox20].

\section{Cursos virtuales}

\section{El Curso de Entornos Virtuales de Aprendizaje de Google (EVAG)}

Estos cursos prepararon a los docentes para impartir educación virtual mediante sesiones síncronas (Meet) y asíncronas (Classroom), iniciando así la transformación a Profesor Digital. Nivel I. Manejo básico de la plataforma G Suite: Meet + Classroom. Nivel II. Gestión de tareas y aplicación de las mejores prácticas en G Suite: Classroom + Formulario. Nivel III. Manejo integral de la plataforma siguiendo las mejores prácticas y competencia para desempeñarse como facilitador y mentor en EVA/G. (Fuente: Profesor Digital 2030-UNMSM. https: //docs.google.com/document/d/1SkvYVhSPXUXkKpu_ 62MtKQiPX40uffmlmPchYMl jpl8/edit)

\section{El Curso de Contenidos Digitales de Google (CEDG)}

Es el complemento del EVA/G y abordó lo referente a contenidos y estrategias para integrar G Suite en el aula. Empodera al docente para facilitar e inspirar el aprendizaje y la creatividad, así como ahorrar tiempo e incrementar su efectividad. Nivel I. Manejo inicial de las aplicaciones para producir contenidos básicos, como documentos, presentaciones, hojas de cálculo, vídeos y Web sites. Nivel II. Manejo avanzado de las aplicaciones para producir contenidos sofisticados, como documentos, presentaciones, hojas de cálculo, vídeos y Web sites. Nivel III. Consolidación del manejo de las aplicaciones para generar contenidos, así como, afianzar su competencia para desempeñarse como facilitador y mentor. (Fuente: Profesor Digital 2030-UNMSM. https: 
//docs.google.com/document/d/1SkvYVhSPXUXkKpu_ 62MtKQiPX40uffmlmPchYMl jp18/edit)

\section{Demostraciones en vídeo experimentales}

Existen páginas de vídeos, uno de ello es la página de YouTube ( https://www.youtube.com/watch?v=Esa3e46Fsg), aquí hay un tutorial a modo de práctica demostrativa de laboratorio de física básica específicamente de Tutorial Caída Libre Tracker Laboratorio de física, además hay otras tales como: manuales, tutoriales, guías, simuladores, prácticas; principalmente para las clases de ciencias básicas, muchos profesores lo han empleado para reforzar sus clases de teoría. Existen páginas exclusivas de vídeo de laboratorio virtuales y paginas exclusivas de prácticas demostrativas. Para el caso de los cursos de física, se han empleado prácticas, tutoriales, ejemplos y modelos de prototipos de proyectos.

\section{Simulaciones de experiencias}

Las simulaciones son demostraciones virtuales de alguna experiencia o algún fenómeno de las ciencias básicas, para los diferentes temas de ciencias físicas. En la internet existen diferentes páginas de simulaciones. Por ejemplo, en física estamos utilizado hasta dos simuladores gratuitos de internet como: El Peth colorado (https:// phet.colorado.edu/es_PE/ ) y el otro es Vazka (https: //www. vascak.cz/physicsanimations.php?l=es), que tienen simulaciones diversas, especialmente para los temas de física. A manera de ejemplo: Para desarrollar la práctica de movimiento de proyectiles utilizamos el simulador (https://phet.colorado.edu/sims/html/ projectile-motion/latest/projectile-motion_es_

PE.html), el profesor muestra el simulador y desarrolla una demostración, luego los alumnos lo experimentan, incluso combinaban los valores iniciales y condiciones, obteniendo sus propios resultados que eran materia de análisis e interpretaciones.

\section{Enseñanza sincrónica}

Es un tipo de enseñanza que se desarrolla en vivo, en directo y en simultáneo entre varias personas o alumnos. En esta etapa de pandemia la educación es virtual, mediante alguna plataforma, esta forma es denominada sincrónica, en dicha forma se ha impartido la enseñanza teórica y práctica a los alumnos desde las aulas virtuales. Por ejemplo para la enseñanza de la Física se explica en tiempo real los conceptos, teorías, fórmulas, leyes, principios de diversos temas de la Física. Así como Schullo, Siekman and Szydlo (2003) "señalan como principal ventaja de los sistemas síncronos de enseñanza y comunicación su alto parecido a una clase presencial. Comparten algunas de sus características definitorias, puesto que facilita el refuerzo inmediato del estudiante, su orientación y guía en el aprendizaje y, por tanto, su motivación en el estudio. Los autores, destacan las innovaciones técnicas que se están desarrollando y que consideran mejoran enormemente las posibilidades de interactividad, así como de expresión de emociones (levantar la mano, reír, aplaudir...). Una de las principales ventajas del uso de sistemas síncronos es la posibilidad de realizar una evaluación previa de los conocimientos que posee el estudiante para posteriormente realizar una adaptación del material del curso a las necesidades de los alumnos y la disciplina. Por otra parte, los autores destacan que este sistema de comunicación puede proporcionar mayor motivación externa al facilitar una interacción más directa entre los usuarios del curso que con los sistemas de comunicación asíncrona. Estos aspectos, concluyen los autores, puede llegar a disminuir la tasa de abandono" [Santoveña12]

\section{Enseñanza asíncrónica}

La enseñanza asincrónica es aquella que se desarrolla estando desconectado, donde cada alumno en forma individual o grupal desarrolla una tarea asignada. Por ejemplo, en nuestra realidad, para los cursos de ciencias básicas, se dejaban tareas o prácticas para que los alumnos trabajen grupal o individual asincrónicamente, luego en el pleno desarrollan en una exposición de su respectivo trabajo. "En el caso de los canales asincrónicos, el proceso de comunicación entre usuarios ocurre sin necesidad de coincidencias espacio-temporales, y, en su gran mayoría son de uso libre y gratuito. Entre ellos, se encuentran: el correo electrónico, listas de distribución, grupos de noticias, foros, transferencia de ficheros y entornos de trabajo colaborativo, como Wikis y Blogs (Barroso y Llorente, 2006)" [Rios17].

\section{Métodos}

Según Sampieri en su libro "Metodología de la Investigación, menciona que el enfoque cualitativo utiliza la recolección y análisis de los datos para afinar las preguntas de investigación o revelar nuevas interrogantes en el proceso de interpretación." [Samp14]. Luego define el "Estudio descriptivo busca especificar propiedades y características importantes de cualquier fenómeno que se analice. Describe tendencias de un grupo o población." [Samp14].

El método de investigación del presente trabajo es descriptivo cualitativo, con manejo estadístico, para determinar la percepción de los estudiantes ante la enseñanza virtual y el uso de herramientas y simuladores de laboratorios de las Facultades de Ciencias como Ciencias 
Físicas y Ciencias Biológicas, además de la Escuela Profesional de Educación y la Escuela Profesional de Ingeniería Eléctrica. Se recolectó información mediante una ficha de encuesta a través de los medios electrónicos, para medir diversos factores relacionados con las capacitaciones, equipos técnicos e internet, docentes y su metodología aplicada y los recursos didácticos.

\section{Instrumento}

El instrumento aplicado es un formulario que está compuesto por 16 ítems. Se distribuyó electrónicamente aproximadamente a 100 estudiantes de las Facultades de Ciencias Físicas y Ciencias Biológicas, además de la Escuela Profesional de Educación y la Escuela Profesional de Ingeniería Eléctrica. Para la recolección de la información se aplicó dicho instrumento, los cuales se estructuraron de acuerdo con la determinación de los objetivos y validados en diferentes opciones de respuesta: desde la validación de sí o no, hasta con la inclusión de preguntas cerradas. El instrumento se estructuró en tres partes:

1.- Capacitaciones, equipos técnicos e internet.

2.- Docentes y su metodología aplicada.

3.- Recursos Didácticos

\section{Interpretación de los resultados de la en- cuesta de estudiantes de San Marcos}

Sobre capacitaciones, equipos técnicos e internet

1. ¿Has participado de la capacitaciones organizadas por la Universidad y/o facultad?

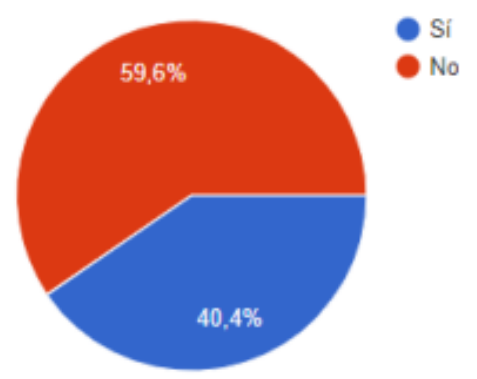

Figura 1: La figura representa el porcentaje de los participantes en las capacitaciones oragnizados por la Universidad

Por medio de comunicación vía correo electrónico, la universidad y sus facultades brindaron capacitaciones; a las cuales asistieron el $40,4 \%$ de los estudiantes encuestados, mientras que el otro $59,6 \%$ dice no haber participado.
2. ¿Cuentas con los equipos apropiados, así como con el servicio de internet para llevar a cabo tus clases virtuales?

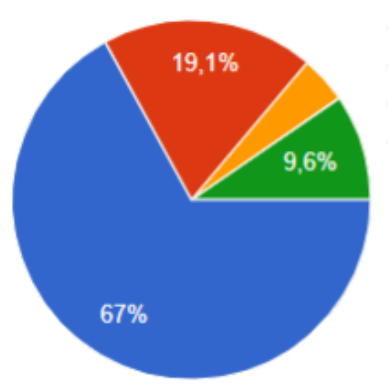

Cuento con equipos e internet

Cuento solo con equipos

Cuento solo con internet

No cuento con equipos, ni con internet

Figura 2: La figura representa el porcentaje de tener objetos electrónicos y a la vez de internet para las clases virtuales

En el caso de la disponibilidad de objetos electrónicos y una conexión de internet, un $67 \%$ de los encuestados afirman poseer estos requerimientos. Un $19 \%$ posee solo equipos, un $4 \%$ cuentan solo con internet y un $9,6 \%$ no posee ninguno de los dos. Esto nos deja ver las dificultades que se pueden presentar a la hora de comunicarse.

3. ¿La Universidad les ha brindado apoyo y asesoría técnica (softwares y/o programas) durante las clases virtuales?

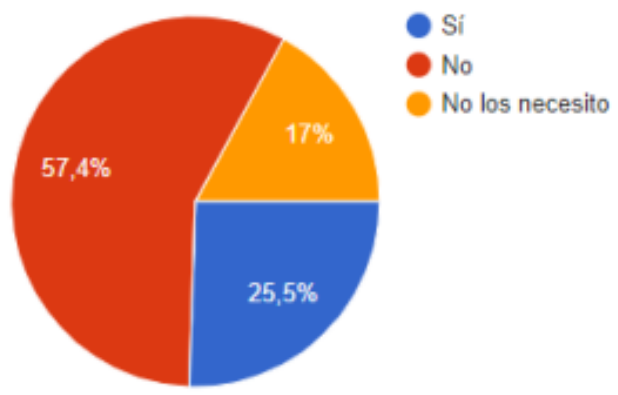

Figura 3: La figura representa los porcentajes de apoyo y asesoria (softwares y/o programas) brindado por la UNMSM

La universidad ha otorgado ciertas asesorías y apoyos sobre programas, como lo afirma un 25,6\% de estudiantes. Para completar los porcentajes, un $17 \%$ dice no haberlo necesitado y el $57,4 \%$ dice 
que no se brindaron tales ayudas y asesorías. Esto sugiere que no hubo una buena comunicación y difusión de estos.

4. ¿Has recibido apoyo de equipos o servicio de Internet para llevar a cabo tus clases virtuales?

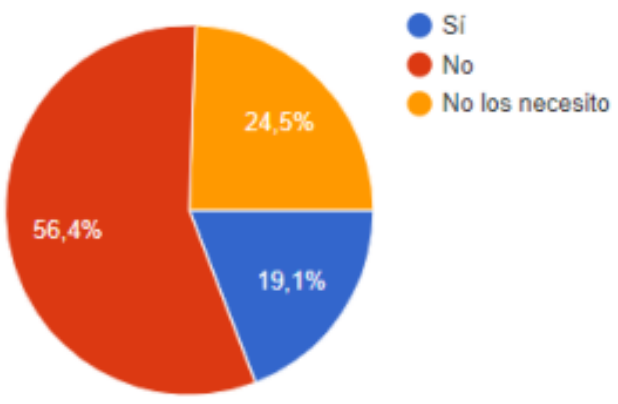

Figura 4: La figura representa el porcentaje de apoyo recibido en equipos o servicio de internet para las clases virtuales

Con respecto al apoyo de equipos o el servicio de internet, un $19,1 \%$ dice haberlo obtenido, un $24,5 \%$ no haberlo necesitado y un $56,4 \%$ no haberlo recibido. Podemos considerar que esto se debe en parte a problemas de las mismas empresas, que no han podido dar un buen servicio y genera la necesidad de obtener este tipo de ayudas.

5. ¿Durante el semestre 2020-I para acceder a la educación virtual. ¿Con qué equipo lo hiciste?

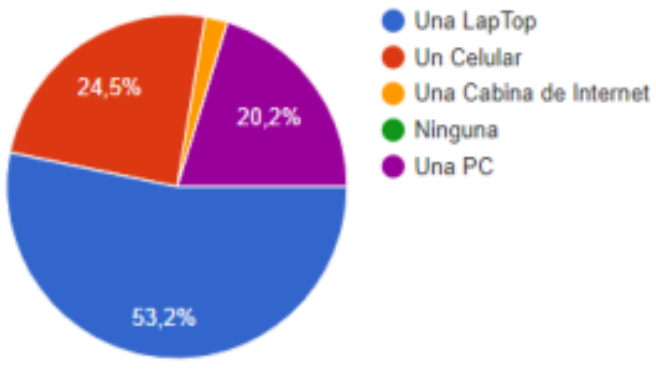

Figura 5: La figura representa el porcentaje del tipo de equipo utilizado para las clases virtuales

Pese a la difícil situación económica por la que la mayoría de familias pasa por la coyuntura actual, la mayoría de encuestados accedió a sus clases virtuales mediante una laptop $(53.2 \%)$, con celular
(24.5\%), con una PC (20.2\%) ello podría evidenciar que dichos estudiantes ya se encontraban preparados o tuvieron que acceder a una laptop, mientras que otros no pudieron afrontar las clases de la misma forma; sin embargo, buscaron otros medios para llevar a cabo las clases.

\section{Sobre los docentes y su metodología}

6. De acuerdo con la experiencia sobre el desarrollo de tus clases virtuales ¿Cómo calificarías la metodología y didáctica de tu docente empleada durante el semestre?

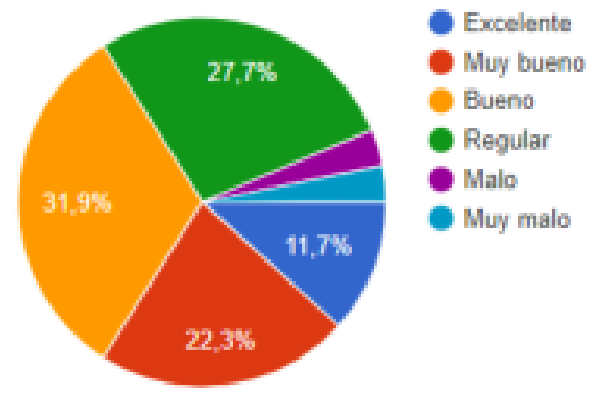

Figura 6: La figura representa el porcentaje de calificación a la metodología y didáctica empleada por el docente

Los estudiantes calificaron como: excelente (11.7\%), muy bueno (22.3\%), bueno (31.9\%) y regular $(27.7 \%)$, la metodología y didáctica docente. Lógicamente, las clases virtuales difieren en muchos aspectos de las clases presenciales, ya que las metodologías se deben adaptar y otros factores como el dominio de la tecnología de algunos profesores y alumnos, así como las dificultades de carácter tecnológico pueden influir negativamente a la calidad de enseñanza en general.

7. En el presente semestre, i. El proceso de enseñanza virtual cumplió con los objetivos del sílabo?

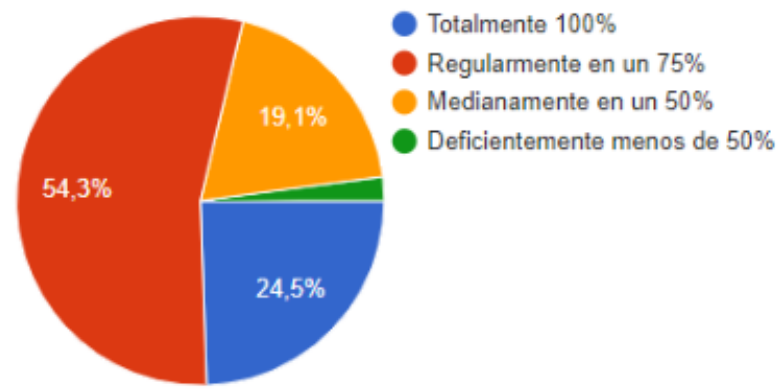

Figura 7: La figura representa el porcentaje del cumplimiento de los objetivos del sílabo 
Los estudiantes manifestaron respecto al cumplimiento de los objetivos del sílabo: totalmente $(24.5 \%)$, regular $(54.3 \%)$ y medianamente (19.1\%); pudo haber sido por por la enseñanza virtual, el uso de las plataformas y las herramientas digitales posiblemente complicó en la enseñanza, ya que el uso de la tecnología para algunos profesores, suelen haberlo hecho con dificultad el proceso de enseñanza.

8. De acuerdo con tu esfuerzo empleado y ponderación ¿Como calificarías el sistema de evaluación empleado?

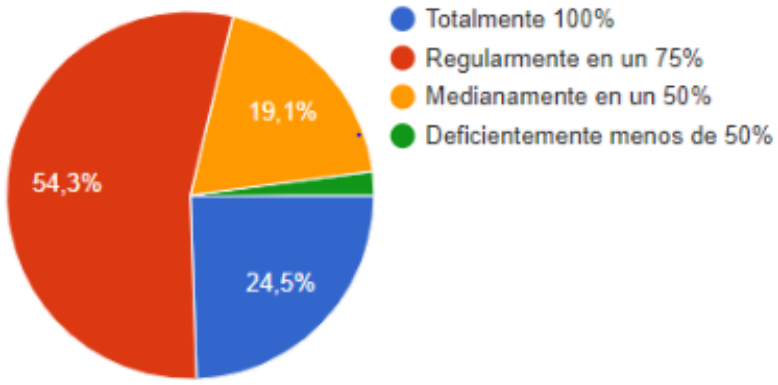

Figura 8: La figura representa el porcentaje de calificación al sistema de evaluación utilizado

Respecto al sistema de evaluación, los estudiantes manifestaron: Totalmente de acuerdo $(24.5 \%)$, regularmente de acuerdo (54.3\%) y medianamente de acuerdo (19.1\%) esto pudo deberse al énfasis en la modalidad actual de la evaluación continua, esto pudo beneficiar a los alumnos que clase por clase van mostrando un avance.

9. De acuerdo con los recursos didácticos empleados en clase ¿Te encuentras satisfecho con los materiales, softwares y simuladores empleados por el docente?

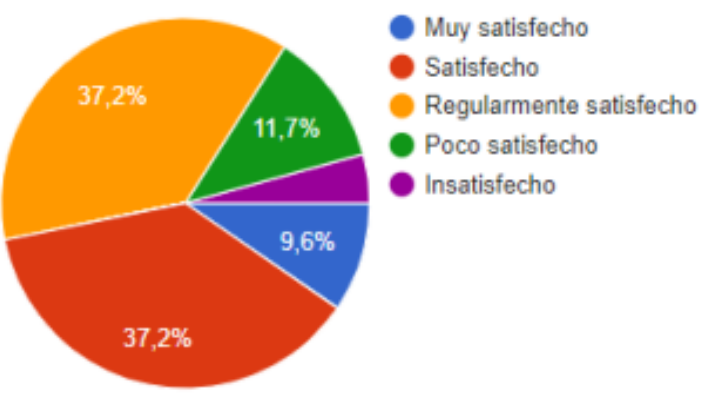

Figura 9: La figura representa el porcentaje de satisfacción con los materiales, softwares y simuladores empleados por el docente
Los estudiantes ante la pregunta si se encuentran satisfechos con los materiales, softwares y simuladores empleados, manifestaron como: muy satisfecho (9.6\%), satisfecho (37.2\%), regular (37.2\%) y poco satisfecho (11.7\%). Muchos docentes se vieron en la necesidad de utilizar diversos tipos de softwares, simuladores y herramientas digitales para reforzar las clase virtuales de sus cursos de ciencias físicas.

10. De acuerdo con su rol y desempeño ¿Cómo calificarías a tu docente en esta etapa de la educación virtual?

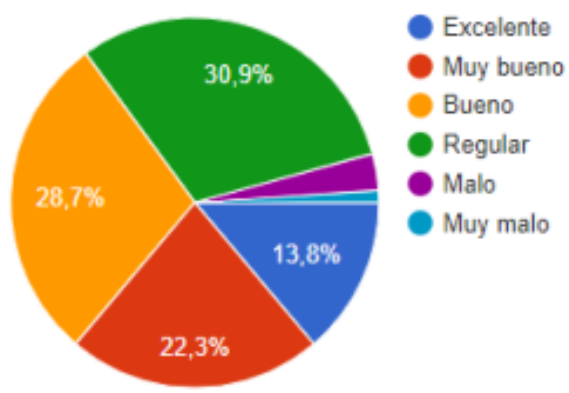

Figura 10: la figura reporesenta el porcentaje de la calificación al docente en esta etapa de la educación virtual

Los estudiantes ante la pregunta como calificaría al docente en esta etapa de la educación virtual, manifestaron como: excelente (13.8\%), bueno (28.7\%), muy bueno (22.3\%), regular $(30.9 \%)$. Hubo un buen desempeño de los profesores de las cursos de ciencias físicas, el uso de sus simuladores y herramientas digitales apropiados para el desarrollo de sus temas se refleja en la calificacion obtenidas de los estudiantes.

11. ¿En qué nivel (porcentaje) crees que la Educación Virtual ha reemplazado a la Educación Presencial?

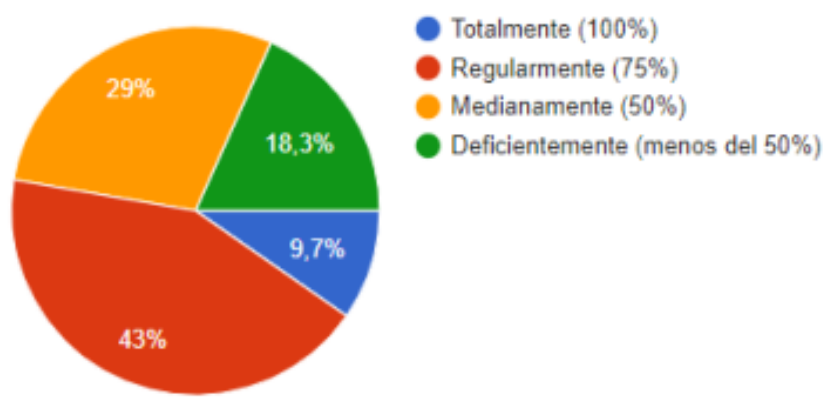

Figura 11: La figura representa el porcentaje de reemplazo de la educación virtual a la educación presencial 
El $9.7 \%$ de los estudiantes encuestado han manifestado que la educación virtual ha reemplazado a la educación presencial, el $18.3 \%$ lo ha reemplazdo deficientemente, un $29 \%$ manifiesta que lo ha reemplzado medianmente y un $43 \%$ manifiesta que lo ha reemplazdo regularmente

\section{Recursos didácticos}

12. ¿La universidad y/o facultad ofrecen(n) recursos y materiales didácticos para el desarrollo de tus clases?

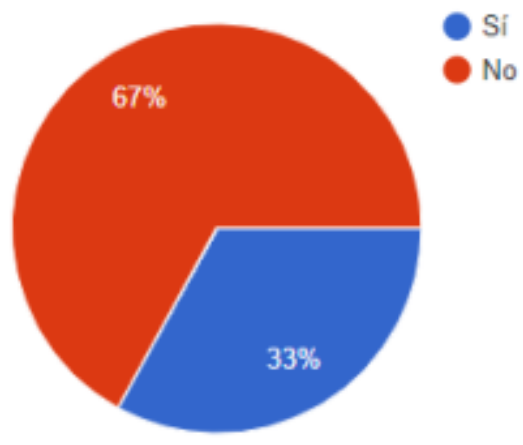

Figura 12: La figura representa el porcentaje de ofrecimiento de recursos y materiales didácticos pare el desarrollo de las clases

El $67 \%$ de personas encuestadas manifiestan que la Universidad Nacional Mayor de San Marcos no ofrece recursos y materiales didácticos, esto puede deberse a que ciertas asignaturas requieren de recursos virtuales que necesitan de algún tipo de suscripción. Sin embargo, la Unidad de la Biblioteca programa constantemente capacitaciones para repositorios bibliográficos, de donde se pueden extraer muchos documentos e información para enriquecer el proceso de auto aprendizaje.

13. En función al desarrollo de tus clases ¿Tus docentes emplean recursos y materiales didácticos en sus cursos (como softwares, simuladores, programas, etc.)?

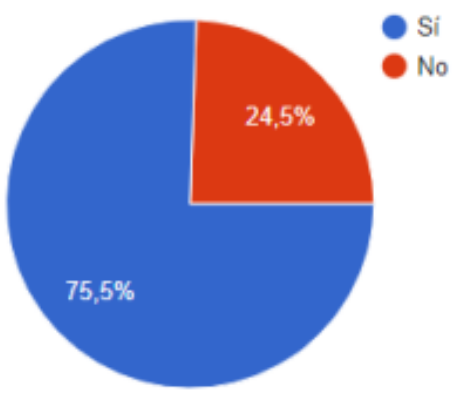

Figura 13: La figura representa el porcentaje del uso de recursos y materiales didácticos empleados por los docente durante el desarrollo de las calses

Muchos docentes buscaron y seleccionaron programas que beneficien a sus alumnos y esto lo respalda el $75,5 \%$ de estudiantes encuestados.

14. ¿El profesor domina el uso de los recursos y materiales didácticos para reforzar la clase virtual?

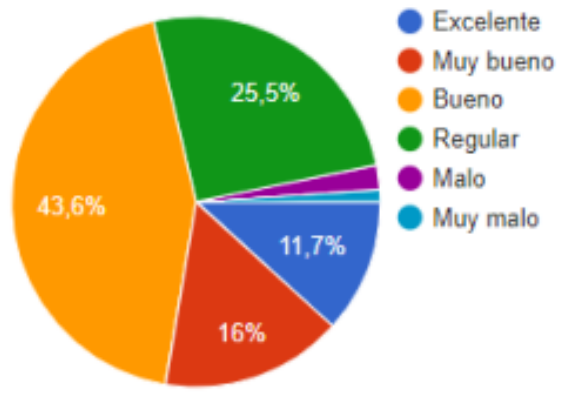

Figura 14: La figura representa el porcentaje del dominio del uso de recursos y materiales didácticos para reforzar la clase virtual

La mayoría, un 43,6\% expresa que sus profesores dominan los materiales didácticos, dejando en evidencia la importancia de las capacitaciones que fueron brindadas. Sin embargo, el esfuerzo y el interés de los profesores para llevar de mejor manera las clases virtuales es decisivo.

15. ¿Los recursos y materiales didácticos sirvieron durante el proceso de enseñanza-aprendizaje virtual?

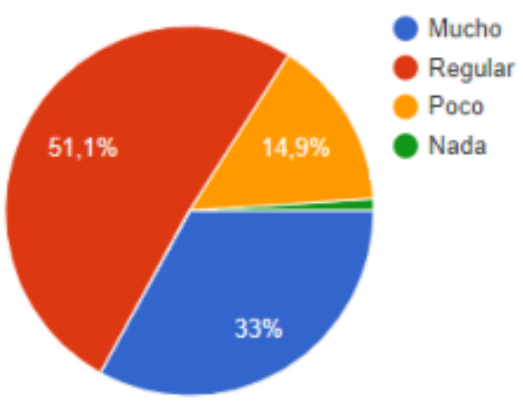

Figura 15: La figura representa el porcentaje de apreciacion de que los recursos y materiales didácticos sirvieron durante el proceso de enseñanza-aprendizaje virtual

El proceso de enseñanza virtual está en constante mejora. Las herramientas empleadas, juegan un papel importante para el correcto desarrollo de este, pues afecta directamente a los estudiantes y a 
su aprendizaje. La mayoría $(51,1 \%)$ opina que estos recursos los ayudaron de manera regular, lo que nos llevaría a buscar o seleccionar nuevos recursos que faciliten este proceso.

16. ¿Cómo calificarías el uso de materiales y recursos didáctico empleados por tu profesor?

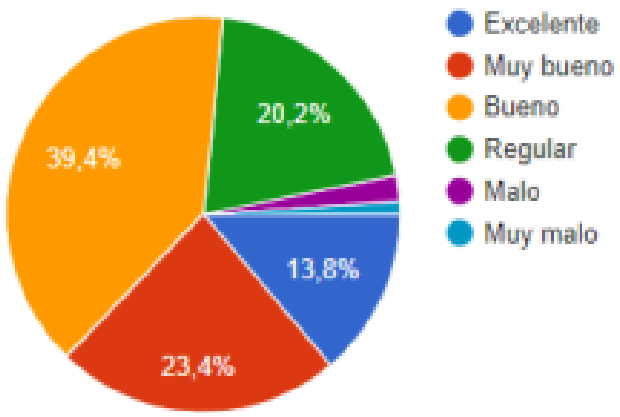

Figura 16: La figura representa el procentaje de calificación en el uso de materiales y recursos didáticos empleados por el profesor

Los docentes se han visto obligados a adaptarse a una educación virtual y para esto hicieron uso de nuevas metodologías y recursos. El 39,4\% considera que sus profesores hicieron un buen uso de ellas, el $23,4 \%$ expresan que fue muy bueno, fue regular para el $20,2 \%$, excelente para el $13,8 \%$ y lamentablemente un $2,1 \%$ y un $1,1 \%$ manifiestan que sus profesores hicieron malo y muy malo el uso, respectivamente, de estas herramientas. Si bien es cierto que los recursos en sí son importantes, también lo es, la aplicación por parte del profesor y debido a esto es que en la Universidad se programaron capacitaciones.

\section{Conclusiones}

Sobre capacitaciones, equipos técnicos e internet

1. La gran mayoría (59.6\%) de los estudiantes encuestados, manifiesta que no ha participado de capacitación alguna de la universidad; para enfrentar las clases virtuales, mientras que el $40.4 \%$ dice que si ha participado de alguna capcitación.

2. La gran mayoría (67\%) de los estudiantes encuestados, manifiesta que cuenta con los equipos y servicios de Internet para llevar a cabo sus clases virtuales, lo que significa que tienen posibilidades de tener un equipo en casa, pero hay un aproximado del $10 \%$ de estudiantes encuestado que manifiesta no tener equipo alguno.
3. El $57.4 \%$ de los estudiantes encuestado manifiesta que no se le ha brindado apoyo ni asesoría técnica respecto a software y/o programas para desarrollar las clases virtuales, mientras que un $25.5 \%$ manifiesta que si se le brindó apoyo y asesoría.

4. El $56.4 \%$ de los estudiantes encuestados manifiesta no haber recibido apoyo de equipos o servicios de internet para llevar acabo de sus clases virtuales, mientras que un pequeño grupo $(19.1 \%)$ manifiesta que si recibió ayuda de la Universidad Nacional Mayor de San Marcos.

5. La gran mayoría de estudiantes encuestados ha manifestado que para llevar su educación virtual ha utilizado una laptop (53.2\%), una PC (24.5\%), un celular $(20.2 \%)$, en esa proporción, existe un grupo de estudiantes $(2.1 \%$ ) que han empleado cabinas de internet, para escuchar sus clases, desarrollar y entregar sus trabajos.

\section{Sobre los docentes y su metodología}

6. Los estudiantes encuestados han manifestado que la metodología y didáctica empleada por los docentes durante el desarrollo de las clases virtuales, lo califican como excelente (11.7\%), muy bueno $(22.3 \%)$, bueno $(31.9 \%)$, aunque un porcentaje significativo también los calificó como regulares $(27.7 \%)$.

7. Los estudiantes encuestados han manisfestados respecto al cumplimiento de los objetivos del sílabo que se cumplió: totalmente $(24.5 \%)$, regularmente $(54.3 \%)$ y medianamente $(19.1 \%)$. Por lo expresado se evidencia que no se pudo completar todos los temas del sílabo.

8. Los estudiantes encuestados han manifestado respecto al sistema evaluación empleado: regularmente $(54.3 \%)$, totalmente $(24.5 \%)$ y medianmente (19.1\%) empleado a través de las clases virtuales. Evidencia un nivel de satisfacción de los estudiantes.

9. Los estudiantes encuestados han manifestado frente a la pregunta de que, si se encuentran satisfechos con los materiales, softwares y simuladores empleados por el profesor durante la clase, se encuentran muy satisfecho (9.6\%), satisfecho (37.2\%) y regularmente satisfecho $(37.2 \%)$ y poco satisfecho (11.1\%).

10. os estudiantes encuestados respecto a la pregunta de cómo calificarías a tu docente durante la etapa de la educación virtual, manifestaron: regular 
(30.9\%), bueno $(28.7 \%)$, muy bueno $(22.3 \%)$ y excelente $(13.8 \%)$, fue un reto de todos los profesores de la Universidad Nacional Mayor de San Marcos.

11. Los estudiantes encuestados respecto a que si la educación virtual ha reemplazado la educación presencial,han manifestado que los reemplazo regularmente $(43 \%)$, medianamente $(29 \%)$, deficientemente $(18.3 \%)$ y un $(9.7 \%)$ piensa que fue reemplazado totalmente, no tiene comparacion las clase presenciales frente a las clase virtuales, salvo algunos cursos teóricos.

\section{Recursos didácticos}

12. Los estudiantes encuestados frente a la pregunta, si la universidad y/o facultades ofrecen recursos y materiales didácticos para el desarrollo de las clases, los estudiantes manifestaron que no (67\%), mientras (33\%) manifiesta que sí. Sin embargo las Facultades y/o Escuelas Profesionales si cuentan con bibliotecas virtuales y digitales.

13. Los estudiantes encuestados fente a la pregunta, si los docentes emplean recursos y materiales didácticos en sus curso como: softwares, simuladores, programas, etc., los estudiantes manifestaron mayoritariamente que si emplearon $(75.5 \%)$, mientras que no emplearon $(24.5 \%)$. Dada la nueva educación se tuvo que recurrir a los recursos apropiados para el desarrollo de las clases.

14. Los estudiantes encuestados frente a la pregunta, si el profesor domina el uso de los recursos y materiales didácticos para reforzar la clase virtual, los estudiantes manifestaron como: excelente (11.7\%), muy bueno (16\%), bueno (43.6\%) y regular $(25.5 \%)$. Lo que se evidencia que los profesores dominan el uso de los recursos y materiales didácticos paa el desarrollo de sus clase virtuales.

15. Los estudiantes encuestados frente a la pregunta, si los recursos y materiales didácticos sirvieron durante el proceso de enseñanza-aprendizaje virtual, los estudiantes manifestaron que le sirvió mucho $(33 \%)$, regular $(51.1 \%)$ y poco $(14.9 \%)$. Esto demuestra la importancia de emplear las herramientas e instrumentos de acompañamiento para las clases.

16. Los estudiantes encuestados frente a la pregunta, cómo calificarías el uso de materiales y recursos didácticos empleados por el profesor, los estudiantes manifestaron como: excelente (13.8\%), muy bueno (23.4\%), bueno(39.4\%) y regular $(20.2 \%)$.Lo que evidencia el conocimineto y uso de stos recursos ara las clase virtuales.

\section{Referencias}

[Fox20] Fox, M., Werth, A., Hoehn, J. and Lewandowski, H. (2020). Teaching labs during a pandemic: Lessons from Spring 2020 and an outlook for the future. Department of Physics, University of Colorado, National Institute of Standards and Technology and University of Colorado, USA.

[Santoveña12] Santoveña, S. (2012). El proceso de enseñanza-aprendizaje a través de herramientas de comunicación síncrona: El caso de Elluminate Live
Electronic Journal of Research in Educational Psychology, vol. 10, No. 1, pp. 447-474.

[Rios17] Ríos, M. y Rodríguez, M. (2017). Comunicación Asincrónica Experiencias en Ambientes Virtuales de Pre y Postgrado. Edición Especial Revista Proyección. Universidad Central de Venezuela. https: //ww .researchgate.net/publication/329362250

[Samp14] Hernández, R., Fernández, C., Baptista, P. (2014). Metodología de la Investigación. Sexta Edición. Editorial McGraw-Hill/Interamericana Editores, S.A. de C.V. México. ISBN: 978-1-4562-2396-0 